\title{
An estimation of gas emissions in Korea's air transport industry
}

\author{
Min-Jung Kim, ${ }^{*}$ Seock-Jin Hong ${ }^{* *}$ and Hun-Koo Ha***
}

\begin{abstract}
This study estimated greenhouse gas emissions from aviation transportation and sought systems that could manage these emissions based on the IPCC guidelines to prepare for greenhouse gas regulations on international airlines. For this purpose, policies to reduce greenhouse gas emissions from aviation transportation were developed based on international agreements and the cases of advanced countries. In addition, marginal abatement costs and greenhouse gas reduction measures were derived for the effective execution of these policies. While estimating greenhouse gas emissions from aviation transportation, it was found that there has been an average increase of $3.9 \%$ and $12.9 \%$ for domestic and international flights, indicating that it is urgent that we prepare global greenhouse gas regulations. The estimated marginal abatement cost of greenhouse gas from airplanes was approximately. USD 123, and this amount could be used to decide the price of emission rights, the amount of carbon tax, and could be referred to when distributing incentives for voluntary agreements.

The measures to reduce greenhouse gas emissions for aviation transportation were classified into four types: voluntary agreements, international collaboration, greenhouse gas reduction technology and operation process development, and application of emission trading and carbon tax.
\end{abstract}

Keywords: greenhouse gas; emission rights; IPCC guidelines; marginal abatement costs; aviation transportation industry

Submission Date: 09/10/2010_Revision Date: 12/10/2010_Acceptance Date: 12/10/2010

* Associate Research Fellow, The Korea Transport Institute, email : mjkim@koti.re.kr

** Professor, Bordeux Management School, email : seokjin.hong@bem.edu

*** Corresponding Author, Associate Professor, Asia-Pacific School of Logistics, Inha University, email : hkha@inha.ac.kr 


\section{Introduction}

The United Nations Framework Convention on Climate Change (UNFCCC) was adopted and officially validated in 1994, followed by the Kyoto Protocol in 1997. Since then, many local and international organizations have actively been discussing the issue of greenhouse gas reduction. The official validation of the Kyoto Protocol from 2005 mandates the Annex I nations to reduce the overall greenhouse gas emission by an average of 5.2\% compared to 1990 during the term of the $1^{\text {st }}$ pledge (2008-2012). Although Korea was classified into non-Annex I and is not obligated to reduce greenhouse gas during the $1^{\text {st }}$ pledge, Korea is expecting to become one of the Annex I nations or set up a spontaneous level of reduction with the Post-Kyoto Protocol to join the global effort to reduce greenhouse gas emissions.

The Kyoto Protocol does not currently apply to the international aviation industry, but its application is being discussed by the EU and other international organizations. As the EU, aside from the Kyoto Protocol, has independent greenhouse gas regulations for airplanes that operate in its territories, subsequent measures are necessary. In order to respond to the rapidly changing greenhouse gas trends of the world, we need to analyze our current level of emission, potential reduction, abatement costs, and administration measures. However, almost no analysis is being done. This study was conducted to rate the level of emission gas from the aviation industry in accordance with the IPCC guidelines, discuss a relevant management system, develop policies to reduce greenhouse gas emissions from aviation transportation based on international agreements and cases from advanced countries, and derive abatement costs and measures for the effective execution of these policies.

\section{Literature Review}

For the purpose of this study, prior research on emission freducing transportation measures (in accordance with the UNFCCC), aviation transportation, and key information on emission statistics systems were analyzed and discussed. For emission ofreducing transportation measures in accordance with the UNFCCC, ${ }^{\circledR}$ Analysis of Effects of Greenhouse Gas Reduction Policies for Transportation Measures in Accordance to UNFCCC(Ministry of Construction and Transportation, 2005, 2006)』discusses the international trends that are reflected on the UNFCCC and the Kyoto Protocol with the potential to impact our economy and transportation in the future and quantitatively analyzes the effects of government policies in relation to energy use requirements and transportation measures.

This part involved an understanding of international trends after the UNFCCC, energy consumption and greenhouse gas emissions in transportation; a pilot study for the analysis of carbon dioxide emissions decision factors to anticipate future carbon dioxide emissions; and an analysis of carbon dioxide emissions reduction based on greenhouse gas reduction 
policies for transportation measures of other countries. In detail, the current study analyzed carbon dioxide emission reduction of 13 transportation policies in three areas-consumption, transportation efficiency improvement, and fuel improvement-for greenhouse gas emissions reduction. However, aviation transportation is different from land transportation and additional models and systems shall be analyzed to suggest reduction policies for aviation transportation.

For reduction policies for aviation transportation in accordance to the UNFCCC, ${ }^{\mathbb{R}}$ Response to EU's Emission Trading System for Aviation Industry (Ministry of Construction and Transportation, 2008)』was conducted to analyze the impact of the EU's emissions trading system and to respond to it as the EU Working Committee had submitted the outlines of the emission trading system that would apply to all airplanes that arrive at and depart from any airport in the EU territories. This part analyzed the EU's execution of the emissions trading system, its expected impacts, the responses of other countries, and its impact on Korea to discuss Korea's possible purchase of emissions, impacts on air fares and domestic airlines, and policies to reduce emission gas.

We discovered we need to build systems to practice greenhouse gas emissions activities, measure and inspect the actual amounts of emissions in order to respond to various emission gas reduction policies for aviation transportation, including the EU's emission trading system. Also, the actual amounts of emissions were calculated based on the constantly discussed allocation of greenhouse gas emissions for international airlines to suggest an emission simulation model with an advantageous allocation for Korea. For the key subject matter of emissions statistics calculation and management, National Strategic Roadmap and Optimized Operation for National Greenhouse Gas Emission Statistics D/B (The Office for Government Policy Coordination, 2006)』analyzed the greenhouse gas emission statistics currently prepared by each government office and derived problems to propose a long-term roadmap to prepare emission statistics and establish preparation systems that match those of advanced countries. In this part, national greenhouse gas emissions statistics were classified into local and international areas and analyzed. Then, the statistics were classified into each industry: energy, environment, agriculture, livestock farming, and forestry to suggest current statistics resources, improvement measures, and a statistics roadmap for the future. Also, a generalized measure and roadmap were suggested for national greenhouse gas emissions statistics preparation.

In conclusion, it was found that Korea's current greenhouse gas emissions statistics system is rather underdeveloped based on the requirements of the Kyoto Protocol. For this reason, this study suggests establishing government offices and/or organizations to steer national greenhouse gas emissions statistics and encourage their collaboration with a memorandum of understanding (MOU). It also proposes that it is necessary to develop emissions factors for each industry and establish systems to evaluate the accuracy of emission statistics. However, this study is limited as its solutions are not specific to the transportation industry, especially aviation transportation. 


\section{Intemational Trends and Responses in the Aviation Transportation Industry}

Each country deploys combinations of various policies to reduce greenhouse gas emissions. Advanced countries are actively investigating the possibility of investing in emissions trading abroad for cost-effective emissions and increasing thee consumption and efficiency of clean energy, such as new regenerative energy in local areas. Certain countries such as Sweden and Denmark are encouraging fewer carbon dioxide emissions and the use of fossil fuels using tax policies, but carbon tax is not currently imposed due to social resistance and effectiveness issues. Each country is highly interested in the UNFCCC, but their response strategies vary according to economic situations and circumstances. As the stances and strategies of countries that are playing key roles in the UNFCCC can contribute to Korea's strategies, we examined the EU and Japan, which are actively reducing greenhouse gas emissions and the U.S., which is not accepting its mandatory obligation to reduce emissions.

\subsection{The U.S.}

The U.S. is superficially confronting the EU in terms of the UNFCCC as it has rejected ratification of the Kyoto Protocol and Former President Bush announced refusal of the Kyoto Protocol. Although the U.S. has been assigned to reduce greenhouse gas emissions by $5 \%$ compared to 1990 , this amount of reduction is equivalent to $30 \%$ reduction based on BAU of 2010. Unlike the EU, which has blunted the increase in energy consumption, the U.S. is seeing a constant increase in energy consumption. The U.S. insists that the Kyoto Protocol would cause 4,900,000 Americans to lose their jobs and an enormous economic cost of $\$ 400,000,000,000$ USD, which would seriously strike its economic condition. It also points out that although it is unfair to limit the growth of developing countries by enforcing unrealistic reduction of greenhouse gas emissions, it is irresponsible to waive the mandates for certain developing countries that are already taking a large portion of greenhouse gas emissions in the world, such as China and India, and stresses the necessity to share burdens with developing countries. However, the U.S. has domestically set a goal to improve greenhouse gas concentration (emissions/GDP) by $18 \%$ by 2012, reduce petroleum consumption by $20 \%$ within 10 years, and increase the use of regenerative energy from $3 \%$ to $15 \%$. Despite that, the U.S. Federal Government has rejected ratification of the Kyoto Protocol, but several state governments have (independently or in solidarity) promoted various greenhouse gas regulatory programs.

In aviation transportation, the U.S. has already reduced considerable amounts of greenhouse gas emissions by executing the following measures of efficiency:

- The airlines saved approx. USD 1,500,000 in fuel costs in 2006 through the efficient use of ground delay to balance consumption and capacity. 
- With the execution of the AFP (Airspace Flow Program), they saved more than 2,380,000 minutes of delay time in 2006 and saved approx. USD 98,000,000 delay cost during that period. About $20-30 \%$ of this amount is thought to be fuel cost.

- Aviation transportation control facilities installed URET (User Request Evaluation Tool) in 2006 to increase capacity and save fuel.

The California State Government has passed a bill to reduce greenhouse gas emissions by $25 \%$ by 2020 . A total of 29 states compromising $65 \%$ of the U.S. populations) have established goals to reduce greenhouse gas emissions. Energy-related research and development is also active to develop hybrid cars, Carbon Capture and Storage (CCS) technologies, and so on. This means that the attainable measures of the UNFCCC could be supported when the the U.S. Government participates with strong determination. Also, nine eastern states, including New York, have promoted a Regional GHG Initiative (RGGI) for local power generators and derived execution measures in April 2005. Other state governments, including Oregon, Massachusetts, New Hampshire, and New England, are promoting independent carbon dioxide emissions regulations and emissions trading programs.

Globally, six Asian-Pacific nations (the U.S., Korea, Australia, Japan, China, and India) are leading the Asian-Pacific Six-Nation Climate Change Partnership to stress technological collaboration for climate change. Last May, they announced the Post-2012 blueprint, which is an expanded form of the Asian-Pacific Partnership and proposed a convention that invites the 15 major greenhouse gas emitters (AP6+EU: Brazil, Canada, Indonesia, Mexico, Russia, South Africa, Turkey, and Ukraine).

\subsection{Japan}

Japan is following the Kyoto Protocol to achieve its mandatory goal to reduce greenhouse gas emissions by $6 \%$ compared to 1990 until 2010. For this purpose, it has established fundamental policies concerning the symbiosis of the environment and economy, step-by-step approaches to response, sharing responsibilities with interested parties, and collaborating with the international society. However, emissions have increased by 6.5\% since 1990 and Japan has realized that it will be difficult to achieve additional reductions by 2010. Many are proposing stricter regulations in order to achieve the goal.

In June 1998, Japan established the Basic Plans against Global Warming (targeting the year 2010), enacted the Global Warning Measures Promotion Act, and revised the Energy Use Rationalization Act to reduce carbon dioxide emissions by improving energy efficiency. To achieve the reduction goal on the Kyoto Protocol, its first step was to increase the efficiency of energy use in industries, homes, commerce, and transportation to reduce emissions back down to their 1990 levels by 2010. The second step was to suppress emissions by $2 \%$ through technological development and societal system changes, $0.5 \%$ at $\mathrm{CH} 4$ and $\mathrm{N} 2 \mathrm{O}, 0.3-3.7 \%$ by increasing forests and other absorbers, $5.2 \sim 1.8 \%$ through the Kyoto 
Mechanism such as emissions trading, joint execution, and clean development systems, and the remaining $2 \%$ by reducing the use of fluoride compounds. ${ }^{1}$

However, Japan, whose energy efficiency is already the highest in the world, can hardly achieve the goal assigned by the Kyoto Protocol through domestic reductions. Thus, it is increasing investments in CDM to purchase emissions trading from other countries. Despite the domestic difficulties with reduction, Prime Minister Abe has announced that the world needs to reduce greenhouse gas emissions by $50 \%$ by 2050 as global warming prevention is a long-term global plan. Japan's official stance toward the Post-2012 is that the world needs flexible and diversified norms where major emitters, such as the U.S., China, and India, can participate, instead of the existing obligations forced by advanced countries. Also, Japan is actively considering the use of its superior clean energy technologies.

Japan's transportation industry is taking approx. $20 \%$ of its total greenhouse gas (GHG) emissions and its aviation industry takes a relatively small proportion of $4 \%$. With the latest increase in efficiency and development of large-sized airplanes, Japan's CO2 emissions in aviation are approx. 20\%, which is even lower than the world's average.

(1) Overview of Japan's Voluntary Plan

Since it signed the Kyoto Protocol in 1997, Japan initiated a voluntary plan for the aviation industry as a part of its Multi-sectoral Program led by Nippon Keidanren. Its goal was to reduce annual CO2 emissions by 10\% from 1990 to 2010. This voluntary plan was integrated into the Department of Transportation's overall transportation program and later changed into the NGWPP (National Global Warming Prevention Package). An intensity targeting was initially used, but it was amended to an absolute level during the the NGWPP renewal of 2002. The aviation industry had already reached the goal in 2004. As the government demanded a higher aim, local airlines proposed 1.9 CO2-MT, which is equivalent to reducing $\mathrm{CO} 2$ emissions by $15 \%$ from 2005 to 2010 . Japan internally evaluated s that the achievements of its domestic airlines are rather remarkable compared to BAU (Business As Usual), as shown in $<$ Fig $1>$.

1 The Office for Government Policy Coordination· Korea Energy Management Corporation, "Easy Interpretation of the Convention on Climate Change, 2006. 


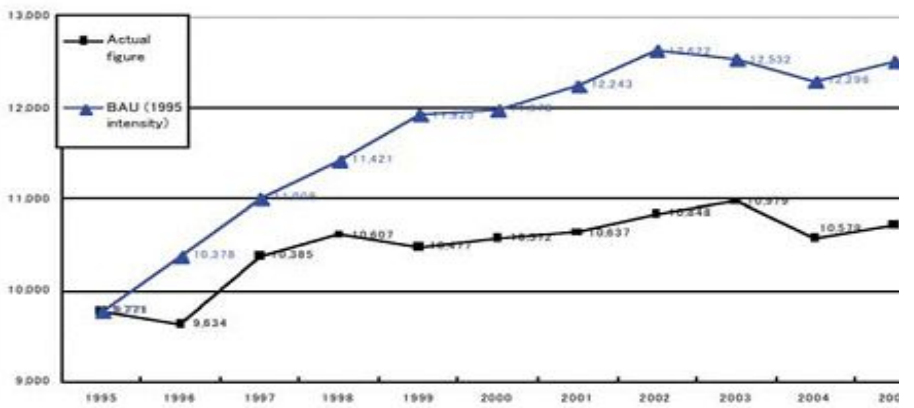

Figure 1.

Domestic Airlines' CO2 Emission before and after Japan’s Voluntary Plan

(2) The Effects of Japan's Voluntary Plan

Japan developed the following formula to analyze the decrease in emissions after its voluntary plan:

$$
\ln \left(\mathrm{CO}_{2} / \text { paxkm }\right)=c+\alpha_{1} t+\alpha_{2} d+\alpha_{3} \ln (\text { dis })+\alpha_{4} L / F+\alpha_{5} \ln (c \cdot p .)+\epsilon
$$

The dependent variable is the number of carbon dioxide emissions for each unit of distance of travel of passenger transportation (RPK), and independent variables are time trends $(\mathrm{t})$, the dummy variable (d) for before and after 1998 to rate the changes after the the voluntary plan, average distance (dis), occupancy rate (L/F), and average size of airplanes (capa).

The results of regression analysis are as shown in $<$ Table $1>$. Efficiency improved by $1.1 \%$ every year from 1985 to 2005 , which is $3.6 \%$ overall since 1998, proving that emissions were significantly reduced by the voluntary plan.

Table 1.

The results of regression analysis

\begin{tabular}{c|c|c|c}
\hline Variables & Estimate Coefficients & Standard of Error & t-statistics \\
\hline \hline $\mathrm{c}$ & 6.90 & 0.35 & $19.45^{* *}$ \\
\hline $\mathrm{t}$ & -0.011 & 0.001 & $-11.44^{* *}$ \\
\hline $\mathrm{d}$ & -0.036 & 0.008 & $-4.52^{* *}$ \\
\hline $\ln (\mathrm{dis})$ & 0.26 & 0.09 & $2.95^{* *}$ \\
\hline $\mathrm{L} / \mathrm{F}$ & -1.24 & 0.06 & $20.98^{* *}$ \\
\hline $\ln ($ c.p. $)$ & -0.49 & 0.07 & $-7.03^{* *}$ \\
\hline rho & -0.37 & 0.20 & -1.90 \\
\hline
\end{tabular}

Source: ICAO, Environmental Report, 2007.

These results imply many significant resources for international aviation transportation policies. 


\section{Marginal Abatement Costs for Greenhouse Gas Emissions Reduction Policies for Aviation Transportation}

\subsection{Greenhouse Gas Emissions Regulation System}

There are various policy measures proposed for a country's greenhouse gas emissions reduction, but they can be classified into direct regulations without any economical induction or economical induction policies using the market mechanism . Direct regulations include greenhouse gas Emission Standards and Technology Standards and policies using the market mechanism including taxation systems (i.e., carbon tax to regulate emissions by changing the costs of greenhouse gas or products), subsidiary systems, and emissions trading systems (ETS) to limit the number of emissions. In addition to these policy measures, the importance of voluntary agreement to reduce greenhouse gas emissions by setting voluntary reduction goals with greenhouse gas emitter businesses has been recognized ${ }^{2}$. The following explains the overview of each policy and mechanism in further detail:

\section{A. Direct Regulations}

Direct regulations involve enacting specific laws to stipulate the standards and acts to be observed by each emitter in order to achieve the desired level of greenhouse gas emissions and by encouraging them to observe laws using various measures and strategies. The Government can establish greenhouse gas emissions standards, technical standards, energy efficiency standards, and various other standards for each emitter. Emission Standards involves having each emitter submit the total amount of emissions allowed and staying below it ${ }^{3}$ and technical standards regulates the technologies and procedures deployed by emitters in economic activities ${ }^{4}$. Direct regulations by setting certain standards are relatively simple and straightforward to adopt and practice compared to other policy measures. Also, they are the easiest to adopt for governments as they can clarify policy goals. However, these regulations bear many problems in terms of efficiency and cost-effectiveness as they limit the choices of emitters by setting up standards instead of using economical induction. Also, direct regulations, except for emission standards, can hardly achieve target emissions by reducing greenhouse gas emissions.

2 Fundamental Study for the 3rd Korean Report for the Convention on Climate Change (Korea Energy Economics Institute, 2004)

${ }^{3}$ Emission standards only indicate the largest possible amount of emission from source, but do not regulate the methods or procedures of emission.

4 For example, obligating the use of exhaust gas filters on cars, regulating the use of certain fuels for sources of electricity, obligating the installation of desulfurizing facilities, etc. 


\section{B. Carbon Tax}

\section{1) Concept}

Carbon tax is the most frequently used policy measure to reduce greenhouse gas emissions. There are two ways to impose carbon tax: First, by imposing taxes on each unit of carbon in fossil fuels, the source of carbon dioxide and second, by imposing taxes on each unit carbon contained in the amount of carbon dioxide emissions from a given source. Carbon tax is related to greenhouse gas emissions and is considered a solution to help suppress carbon dioxide emissions, one of the major greenhouse gases responsible for global warming. Carbon dioxide is generally emitted by the combustion of fossil fuels. Except for partial incomplete combustion, carbon dioxide emissions are determined by the carbon contents of the type of fuel used. As carbon dioxide provides clear numbers for injection and calculation for accurate measuring, carbon tax is considered an appropriate policy measure to reduce carbon dioxide emissions.

The imposition of carbon tax increases the price of fossil fuels, such as petroleum, coal, and gas, to reduce consumption of fossil fuels and carbon dioxide emissions. Also, carbon tax indirectly reduces carbon dioxide emissions in three ways: new industrial structure, technological development, and environmental protection awareness. First, the increase in the price of fossil fuels with the imposition of carbon tax increases the prices of commodities that are produced by intensive use of fossil fuels. This changes industrial structures into low-carbon structures and encourages the consumption of low-carbon products to reduce carbon dioxide emission. Second, carbon tax accelerates the development of energy-saving technologies and increases investment in energy-saving facilities to reduce carbon dioxide emissions. Last, carbon tax improves the citizens' environmental preservation awareness to reduce unnecessary energy consumption and suppress carbon dioxide emissions.

\section{2) Theoretical Background 5}

In order to achieve a socially optimal number of emissions, the rate of carbon tax must correspond to the amount of marginal damage caused by carbon dioxide emissions, regardless of whether carbon tax is imposed on carbon itself or on the number of carbon dioxide emissions ${ }^{6}$. $<$ Fig. $2>$ can be used to illustrate how carbon tax is imposed on a company that uses fossil fuels and emits carbon dioxide.

\footnotetext{
5 The theoretical background of carbon tax is based on the theory of emission duties in the study of Environmental Economics.

${ }^{6}$ It is similar to the Pigouvian Tax, which imposes taxes on each unit of emission up to marginal social damage caused by each polluter to internalize external diseconomies incurred by pollution.
} 


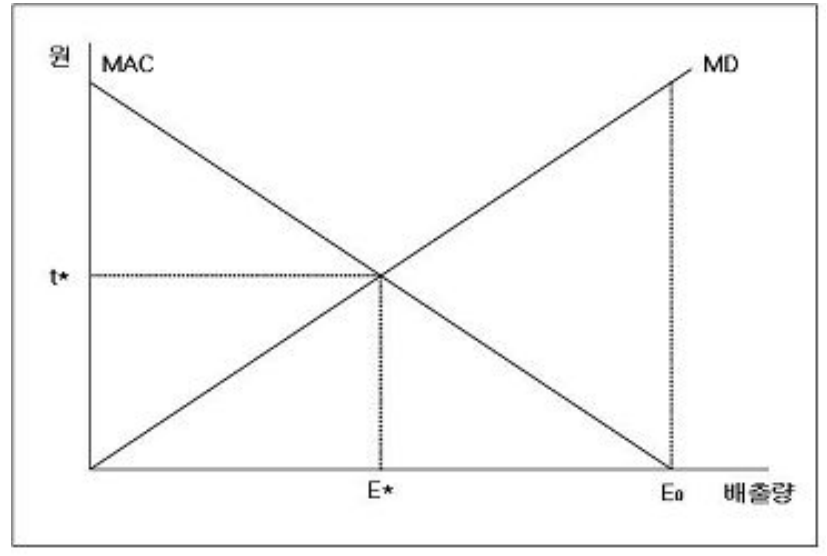

Figure 2.

Optimization of Carbon Tax

As companies do not make efforts to reduce the emission of pollutants until the imposition of carbon tax, the starting number of emissions is set to $E_{0}$. The marginal abatement cost (MAC) curve increases as the number of emissions moves from $E_{0}$ toward the left. It takes little cost to reduce emissions slightly, but it takes large investments or costs (i.e., new technologies or renunciation of business) to decrease emissions by considerable rates.

The increase in the emission of pollutants is followed by an increase in damage. This effect can be illustrated by the marginal damage (MD) curve. The anticipated marginal damage when reducing the emission of pollutants by a unit from $E_{0}$ exceeds marginal abatement cost (MAC), it is possible to increase net social benefit by reducing the amount of emission. The increase in net social benefit with the reduction of emission persists until the amount of emission is decreased down to $E^{x}$. Therefore, $E^{x}$ can be considered the optimal amount of emission that maximizes the level of social welfare and benefit. The amount of carbon tax needed to reduce the amount of emission to an optimal level $\left(E^{x}\right)$ becomes the size of the marginal damage or marginal abatement cost that corresponds to the optimal amount of emission. When carbon tax is imposed in the amount of $t^{x}$, the marginal abatement cost stays lower than the rate of carbon tax until the amount of emission reaches $E^{x}$ and continues to induce low levels of emission. However, when emissions exceed $E^{x}$, the marginal abatement cost surpasses the rate of carbon tax and fails to induce low levels of emission. In addition, carbon tax can also create additional tax sources for the government and such sources of funds can improve the taxation system, increase investment in environmental protection, and alleviate negativities against carbon tax.

Carbon tax is superior to other policy measures in terms of cost-effectiveness and technological development; however, it actually incurs great economic cost by suppressing economic activities and growth, weakening national power, decreasing consumer benefits, 
and causing inequalities in income distribution. When a government decides to impose carbon tax to achieve desired levels of carbon dioxide emissions, it needs to have an accurate layout of the marginal profit curve and marginal cost curve for each source of emission. In other words, the government must be able to have accurate information from each source of emission. The government must be able to record the total amount of emission from each source during a certain period of time through constant measuring of emissions from each course. Such measuring incurs high costs and is realistically impossible. If carbon tax is imposed based on inaccurate information, the initial target amount of emission would be hardly achieved and controlling the overall amount of carbon dioxide emission becomes very difficult. In particular, prevention of global warming, the big purpose underlying carbon tax, is a global issue. Therefore, carbon tax implies global assistance both conceptually and practically?

\section{Emission Trading System 8}

\section{1) The Concept of Emission Trading System}

Emissions trading, which was proposed by political scientist Dales (1968) of the University of Toronto in Canada as a way of converting the shared properties of natural resources into private properties, is manifested by many economists as an economic approach to solve environmental problems along with emissions duties (i.e., carbon tax). In fact, many countries including the U.S. are using, or planning to adopt, this system. In particular, it is recognized as the most well-known policy measure for greenhouse gas reduction in Korea in relation to the UNFCCC. Emissions trading is very similar to the aforementioned carbon tax in terms of efficiency, but its execution and adoption are very different.

Coase (1960) posits that external issues, including environmental issues, are caused by property rights that are unclear or undefined. Emission trading induces voluntary exchanges by clarifying the scopes of property rights. In other words, emission trading defines permissions or rights relating to the environment or natural resources as comparable, quantitative concepts and allows their trading. In the emission trading market, emission demand and supply are decided by market demand and price control. In other words, if an emitter can emit greenhouse gas at an affordable rate, it can decrease its own emissions and sells its emission rights in the market for additional profit. On the other hand, if a high rate of emission is imposed on an emitter, it can purchase emission rights in the market instead of sabotaging its business to reduce emissions. A new emitter can also purchase emission rights to emit greenhouse gasses. In sum, emission trading allows the government to set the

\footnotetext{
7 Lee, 『Study of Environmental Economics』, 2000.

${ }^{8}$ Lee, ${ }^{『 S t u d y ~ o f ~ E n v i r o n m e n t a l ~ E c o n o m i c s 』, ~} 2000$.
} 
desired level of greenhouse gas emission and allot transferrable emission rights for each emitter to emit certain amounts of greenhouse gas, and encourages emitters to trade their rights in the market.

\section{2) Cost-effectiveness of Emission Trading}

$<$ Fig $3>$ illustrates the principle of emission trading. Emission rights of Participant $A$ and Participant $B$ were set to $\left(R_{A}\right)$ and $\left(R_{B}\right)$, respectively, and their marginal abatement costs were set to $\left(M A C_{A}\right)$ and $\left(M A C_{B}\right)$. It was anticipated that $A$ 's marginal abatement cost is larger. The total cost saved by $A$ was $\left(\triangle R_{A} C_{A} 0\right)$ and the total cost saved by $B$ was $\left(R_{B} C_{B} 0\right)$. $A$, with larger marginal abatement cost, endures a relatively high reduction cost.

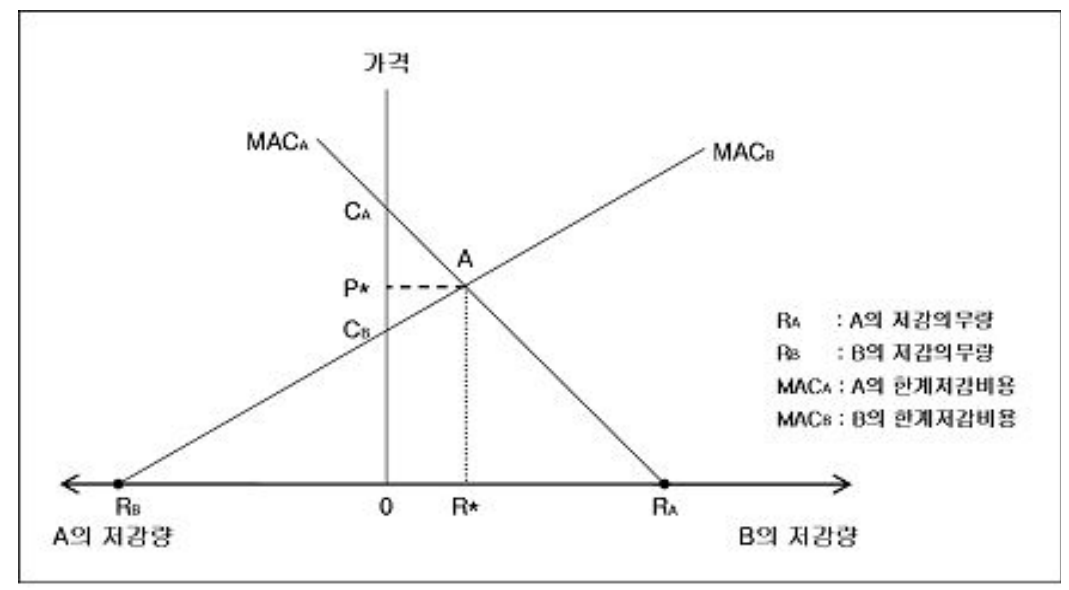

Figure 3.

Economic Benefits of Emission Trading

Under emission trading, participants reduce emission up to the level where they can maximize profits. For this purpose, $A$ and $B$ set the level where their marginal abatement costs correspond to each other. As a result, $A$ reduces less than its initial goal $\left(R_{A}-R^{x}\right)$ and purposes additional emission rights $\left(R^{x}\right)$, whereas $B$ has excess emission rights $\left(R_{B}+R^{x}\right)$ to sell $\left(R^{x}\right)$. In this case, the emission rights $\left(\square A P^{x} 0 R^{x}\right)$ are sold at $\left(P^{x}\right)$. $A$ 's total cost saved is $\left(\triangle A R_{A} R^{x}+\square A P^{x} 0 R^{x}\right)$ and makes $\left(\triangle A C_{A} P^{x}\right)$ of profit by trading emission rights. $B$ saves a total of $\left(\triangle A R_{B} R^{x}-\square A P^{x} 0 R^{x}\right)$ and makes $\left(\triangle A C_{B} P^{x}\right)$ of profit to acquire $\left(\triangle A C_{A} C_{B}\right)$ of social benefits. In other words, emission trading is a policy measure for market economies that induces participants with low 
marginal abatement costs to mit less greenhouse gas by creating cost-saving effects for every participant, and encourages the society to achieve its sales goals as a whole.

\section{Subsidies System}

In this system, greenhouse gas emitters are given rights to emit greenhouse gas up to a certain amount and receive government subsidies when they renounce parts of their rights. Such subsidies come from taxes collected from the general public. The subsidies system is closest to the principle of imposition on beneficiary or victim in terms of greenhouse gas emissions and is largely classified into subsidies for greenhouse gas reduction facilities and for reduction.

Subsidies for reduction facilities are provided when greenhouse gas reducing facilities are installed. The government can pay all or parts of the cost of installation ${ }^{9}$. On the other hand, subsidies for reduction are paid by the government for each unit of emission when a qualified emitter emits less greenhouse gas than the amount it is allowed to emit. For this subsidies system to operate properly, the government that pays the subsidies must have accurate records of information, such as technological capacity, cost, and potential reduction for each qualified beneficiary. Socially desirable cost-effectiveness cannot be achieved if the government fails to gather or interpret such information properly. Also, the government needs to gather funds by imposing taxes to pay for the subsidies. Even if the subsidies can reduce greenhouse gas emissions for efficient distribution of resources in the short-term, the source of funds and taxes distorts the market economy that is already distributing natural resources efficiently ${ }^{10}$. In addition, subsidies can reduce the amount of burden on greenhouse gas emitters and transfer parts of the burden on taxpayers that need to pay additional taxes to fund the subsidies.

\section{E. Voluntary Agreement}

Voluntary Agreement is a non-regulatory policy measure that allows companies, businesses, and governments to discuss and promote greenhouse gas reduction goals, measures, and support voluntarily. It is known as an effective policy measure that can be flexibly adjusted for each type of industry and can develop efficient reduction measures. Because of these advantages, many companies in advanced countries are adopting voluntary agreement to reduce greenhouse gas emissions. The efficiency and cost-effectiveness of this policy can be achieved when participating companies have an accurate understanding

\footnotetext{
9 Subsidies for reduction facility can be used to pursue both industrial protection and greenhouse gas reduction as they can reduce greenhouse gas emission without sacrificing the profitability of emitters. However, they limit choices for emitters as they support only one way of reducing greenhouse gas.

10 If carbon tax is imposed to raise funds, subsidies system can be combined with carbon tax system.
} 
of costs saved and inject minimum costs to achieve the greatest possible reductions. Voluntary agreement has no fairness issue among participating companies as they voluntarily discuss with the government to decide the amount of greenhouse gas reduction, but there still is the fairness issue with companies that are not participating in the voluntary agreement. It may also be an inefficient measure for the country as a whole as participants voluntarily decide the amount of greenhouse gas reduction and there are no penalties even if the target amount of reduction is not met.

\subsection{The Importance of Economic Induction to Reduce Greenhouse Gas and Necessity} to Anticipate Marginal Abatement Cost for the Aviation Transportation Industry

In achieving environmental goals, economic induction systems, such as emission regulations and emission trading, are proven to be more cost-effective than direct regulations. Under direct regulations, regulators must decide the amount of emission allowed for each source of emission based on the socially optimal total amount of emission, but it is difficult to determine the social marginal damage curve and marginal abatement cost curve that relate to pollution and set the maximum amount of emission based on these curves.

As a result, direct regulations could easily lead to incomplete monitoring, supervision, and execution of emission control. Also, it might not be able to minimize social cost as it does not consider the difference in marginal abatement costs of different pollutants generated by each industry. Furthermore, it could face resistance when it is uniformly applied to all industries or when there is no subjective emissions measuring system. On the other hand, the economic induction system gives companies the freedom to decide whether they will emit pollutants based on marginal abatement costs and pay duty, purchase emission rights, or voluntarily reduce pollutants. By doing so, it induces the reduction of emissions with minimal social costs.

For example, if a company's marginal abatement cost of a certain type of pollutant is KRW 1,000,000 per ton and the environmental tax exceeds this amount, it would voluntarily choose to reduce pollutants, and by doing so saves cost than paying the tax. On the other hand, if the environmental tax is less than KRW 1,000,000 per ton, it would pay the tax to increase profits. For this mechanism to operate, it is necessary to have accurate information on companies' emission of pollutants and marginal abatement costs. However, the aviation transportation industry is still lacking an accurate calculation of its gas emissions and research on marginal abatement costs of greenhouse gasses. Therefore, it is difficult to execute the economic induction system. Thus, it is also difficult to promote negotiations for a voluntary agreement. In this respect, the amount of gas emission from aviation transportation in Chapter 3 and the marginal abatement costs of greenhouse gas in the aviation transportation industry, which is discussed in the following section, would be very useful when promoting national policies to reduce greenhouse gas emissions for the post-Kyoto system and contribute to establishing efficient policies. 


\section{Substantial Model and Results of Estimation}

\subsection{Model}

This study used Pittman's Translog Production Function (1981) to estimate the marginal abatement cost of emissions for the following reasons: Pittman's study is significant in that it developed the first production model with awareness that pollutants are emitted as byproducts and estimated the marginal abatement cost of emission using this model. Since Pittman, many studies have used Pittman's resources to compare results. In Korea, however, Pittman's model has been used for fire power generators, but not for transportation. Cobb-Douglas function and CES (Constant Elasticity of Substitution) are widely used production functions, but are sometimes incompatible with certain situations due to their restrictions. Restricted flexibility for substitution and conversion is extremely limited for models with one or more operations and two or more elements of input. Therefore, we prefer a model that is flexible and without restrictions. In this respect, the Translog Production Function was appropriate as it does not impose restrictions on substitution of elements of input. The Translog Production Function, which includes pollutants as byproducts of production, is expressed as follows:

$$
\begin{aligned}
\ln Q= & \alpha_{0}+\alpha_{x} \ln Z+\sum_{i} \alpha_{i} \ln X_{i} \\
& +1 / 2 \beta_{x x}(\ln Z)^{2}+1 / 2 \sum_{i} \beta_{i i}\left(\ln X_{i}\right)^{2} \\
& +\sum_{i} \gamma_{i j}(\ln Z)\left(\ln X_{i}\right)+1 / 2 \sum_{i} \sum_{j}^{i \neq j} \gamma_{i j}\left(\ln X_{i}\right)\left(\ln X_{j}\right)
\end{aligned}
$$

Here, $Q$ is the product, which stands for revenue ton-km in this study, and $Z$ is the amount of greenhouse gas emission. Also, $X_{i}(i=L, K, E)$ are the elements of input, representing labor, capital, and fuel. $\ln Q$ is expressed as $\left(\gamma_{\xi}, \gamma_{i i}\right)$, the log combination of quadratic functions $\left(\alpha_{x}, \alpha_{i}, \beta_{x x}, \beta_{i i}\right)$ and other operations and elements of input. The condition of balance is $\gamma_{i j}=\gamma_{i j}, i \neq j$ in every case. In the model, undesired greenhouse gas $Z$ is considered a normal element of input $\left(\frac{\vartheta Q}{\vartheta Z}>0\right)$ and is used in the calculation of profit maximization. Now, think about profit maximization of a company that uses an element of input $X_{i}$ under a general production function $Q=f\left(Z, X_{L}, X_{K}, X_{E}\right)$ to produce two products $Q$ and $Z$. Here, the price of element of input $X_{i}(i=L, K, E)$ is $P_{i}(i=L, K, E)$. The nominal price of product $Z$ does not exist, but can be found in the process of solving the problem of profit maximization. In the case of undesired product $Z$, each individual producer cannot consume more than the designated amount $\left(Z^{x}\right)$ and would pursue profit maximization within the designated amount. Therefore, the issue of profit 
maximization is expressed as follows:

$$
\begin{array}{ll}
\operatorname{Max} & P_{Q} \cdot Q-\sum P_{i} X_{i} \\
s \cdot t & Q=f\left(Z, X_{L}, X_{K}, X_{E}\right) \\
& Z^{x} \geq Z
\end{array}
$$

The Lagrange Function to solve the above problem of profit maximization is shown as follows:

$$
\Psi=P_{Q}(Q) \cdot Q-\sum P_{i} X_{i}+\lambda_{1} f\left(Z, X_{1}, \cdots, X_{5}\right)-Q+\lambda_{2}\left(Z^{x}-Z\right)
$$

Here, $\lambda_{1}$ is the shadow price of production function restrictions and $\lambda_{2}$ is the shadow price of greenhouse gas restrictions. The marginal abatement cost of greenhouse gas is derived through production function based on the relationship between emission, product, and production elements and the marginal abatement cost itself is estimated based on changes in profits according to changes in emission. In other words, calculations and production elements are changed to change emissions and total profit changes are calculated to measure the marginal abatement cost. When the above formula undergoes partial differentiation for products, greenhouse gas, and each element of input in order to find Condition 1 of Formula (2), the following relations are found:

$$
\begin{aligned}
& \frac{\partial \Psi}{\partial Q}=P_{Q}+Q \frac{\partial P_{Q}}{\partial Q}-\lambda_{1}=0 \\
& \frac{\partial \Psi}{\partial Z}=\lambda_{1} f_{2}-\lambda_{2}=0 \\
& \frac{\partial \Psi}{\partial X_{i}}=P_{i}+\lambda_{1} f_{i}=0(\text { for each } i)
\end{aligned}
$$

Now, Condition 1 can be organized for profit maximization to find the following relations:

$$
\begin{aligned}
& f_{2} \cdot P_{Q}\left(1-\frac{1}{n}\right)=\lambda_{2}(3 a) \\
& f_{i} \cdot P_{Q}\left(1-\frac{1}{n}\right)=P_{i}(3 b)
\end{aligned}
$$

Here, $n$ is the absolute value of product $Q$ 's elasticity of demand $\left(n=\left|\frac{d \ln Q}{d \ln P}\right|\right)$. Formulas (3a) and (3b) manifest that marginal profits of input $\mathrm{s}$ correspond to the prices of inputs. Formulas (3a) and (3b) can be solved for $f_{2}$ and $f_{i}$ as follows: 


$$
\begin{gathered}
f_{2}=\frac{\lambda_{2}}{P_{Q}\left(1-\frac{1}{n}\right)(4 a)} \\
f_{i}=\frac{P_{i}}{P_{Q}\left(1-\frac{1}{n}\right)(4 b)}
\end{gathered}
$$

The following relations can be obtained when each side of Formula (4a) is multiplied by $Z / Q$ and when each side of Formula (4b) is multiplied by $X_{i} / Q$ :

$$
\begin{aligned}
\frac{f_{2} Z}{Q} & =\frac{\lambda_{2} Z}{P_{Q}\left(1-\frac{1}{n}\right) Q(5 a)} \\
\frac{f_{i} X_{i}}{Q} & =\frac{P_{i} X_{i}}{P_{Q}\left(1-\frac{1}{n}\right) Q(5 b)}
\end{aligned}
$$

Generally, $f_{2}=\partial Q / \partial Z, f_{i}=\partial Q / \partial X_{i}$ and the left sides of (5a) and (5b) are as follows:

$$
\begin{aligned}
& \frac{\partial Q}{\partial Z} \cdot \frac{Z}{Q}=\frac{\partial \ln Q}{\partial \ln Z} \\
& \frac{\partial Q}{\partial X_{i}} \cdot \frac{X_{i}}{Q}=\frac{\partial \ln Q}{\partial \ln X}
\end{aligned}
$$

Applying these to (5a) and (5b), the following are obtained:

$$
\begin{aligned}
& \frac{\partial \ln Q}{\partial \ln Z}=\frac{\lambda_{2} Z}{P_{Q}\left(1-\frac{1}{n}\right) Q(6 a)} \\
& \frac{\partial \ln Q}{\partial \ln X_{i}}=\frac{P_{i} X_{i}}{P_{Q}\left(1-\frac{1}{n}\right) Q(6 b)}
\end{aligned}
$$

When Formula (1) is differentiated for $Z$ and $X_{i}$, the following are obtained:

$$
\begin{aligned}
& \frac{\partial \ln Q}{\partial \ln Z}=\alpha_{x}+\beta_{x x} \ln Z+\sum_{i} \gamma_{\xi} \ln X_{i}(7 a) \\
& \frac{\partial \ln Q}{\partial \ln X_{i}}=\alpha_{i} \beta_{i i} \ln X_{i}+\gamma_{\xi} \ln Z+\sum_{i}^{i \neq j} \gamma_{i j} \ln X_{j}(7 b)
\end{aligned}
$$


Formulas (6a) and (6b), and Formulas (7a) and (7b) can lead to the following Formulas (8a) and (8b). As $\lambda_{2}$ is an unknown parameter, it moves to the right side of Formula (8a).

$$
\begin{aligned}
& \frac{Z}{P_{Q}\left(1-\frac{1}{n}\right) Q}=\frac{\alpha_{z}}{\lambda_{2}}+\frac{\beta_{z z}}{\lambda_{2}} \ln Z+\sum_{i} \frac{\gamma_{z i}}{\lambda_{2}} \ln X_{1(8 a)} \\
& \frac{P_{i} X_{i}}{P_{Q}\left(1-\frac{1}{n}\right) Q}=\alpha_{i}+\beta_{i i} \ln X_{i}+\gamma_{\xi} \ln Z+\sum_{j}^{i \neq j} \gamma_{i j} \ln X_{j}(8 b)
\end{aligned}
$$

Formulas (8a) and (8b) are estimated along with Formula (1). When disturbance terms are evenly distributed through all three functions, the iterative Zellner estimation procedure ${ }^{11}$ was used to estimate the Maximum Likelihood Estimator (MLE).

\subsection{Resources}

The current study used the product and input resources of Korean Air from 1991 to 2006 and of Asiana Airlines and Cathay Pacific Airlines from 1991 to 2002. There were 40 elements of observation in total. Cathay Pacific Airlines was included because it could be insufficient and insignificant to consider Korean airlines only and Cathay Pacific Airlines is determined to have a cost structure similar to Korean airlines as it focuses mainly on operating international flights. For the purpose of study, revenue ton-km was set as the product, whereas labor, fuel, capital, and greenhouse gas emission were set as elements of input. Quantity and price data were gathered for each variable. Elements of input and prices of domestic airlines from 1991 to 2002 for the estimation were gathered from 『Study on Restructuring Aviation Transportation Industry through Cost Analysis (The Korea Transport Institute, 2006)』and additional resources were used to gather the data for the Korean Air after 2003 .

Most data for domestic airlines were collected from their 'sales reports,' 'settlement reports,' and 'management performances' and data for Cathay Pacific Airlines were gathered from the balance statement of ICAO 'Financial Data' and 'Fleet \& Personnel'12. For consistency, the same categories were used for the tabulation of data for both domestic airlines and Cathay Pacific. The following are further details on gathering resources for the elements of input. The element of input for labor was the number of employees and that for capital was the

\footnotetext{
${ }^{11}$ The iterative Zellner estimation procedure is used to solve simultaneous equation of two or more equations when all variables are exogenous variables and error terms of each equation seem unrelated, but are actually correlated with one another (A. Zellner, 1962, pp.348 368).

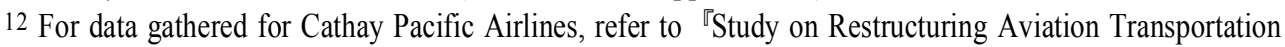
Industry through Cost Analysis』.
} 
number of airplanes. For fuel, fuel consumption had to be the element of input, but revenue ton-km was used as a substitute variable due to limited resources. Also, the estimated value from the previous chapter was used for greenhouse gas emissions, except for Cathay Pacific Airlines' greenhouse gas emissions, which were estimated based on domestic airlines' greenhouse gas emissions per revenue ton-km. The element of input for price was derived by dividing cost by quantity. $<$ Table $2>$ shows the three categories of cost and quantity selected for this study. The cost was converted into U.S. dollars using an annual average exchange rate for comparison with the data of Cathay Pacific Airlines and into the constant price of 2002 using the U.S.' GDP deflator .

Table 2.

Definition of Input variables

\begin{tabular}{c|l|l}
\hline & \multicolumn{1}{|c}{ Factor of Cost } & \multicolumn{1}{c}{ Input } \\
\hline \hline Labor & $\begin{array}{l}\text { Salary, Healthcare, Accommodation for crew, Retirement } \\
\text { and etc. }\end{array}$ & Number of employees \\
\hline Fuel & Fuel cost & Revenue ton-km \\
\hline & $\begin{array}{l}\text { Leasing cost for aircraft including amortization, insurance } \\
\text { fees and damage, Interest rate for fixed asset and } \\
\text { amortization, Airport charge(Landing charge, facilities utility } \\
\text { charge, ground handling charge), Other(Maintenance, Sales } \\
\text { and Marketing, general administrative, etc. }\end{array}$ & Number of aircraft \\
\hline
\end{tabular}

For elasticity of demand, a value from preceded study was used. According to Oum, et al. (1992), the range of estimated price elasticity of demands of aviation passengers is between -0.4 and -4.51 and most values are placed between -0.8 and -2.0 . Therefore, this study used the median, which is -1.5 . For the average price of products, the sales of airlines were divided by the quantity in revenue ton-km <Table $3>$ lists the average values of variables that are included in our production function, including the elements of input, prices, products, and greenhouse gas emissions. 
Table 3.

Average value of variables(As of 2002)

\begin{tabular}{c|c|c}
\hline \multicolumn{1}{c|}{ Input and output } & Average value \\
\hline \hline \multirow{4}{*}{ Input number } & Labor(person) & 12,866 \\
\cline { 2 - 3 } & Fuel(revenue ton-km) & $11,273,900$ \\
\cline { 2 - 3 } & Aircrafts & 87 \\
\cline { 2 - 3 } Input value & Gas emission(ton) & $2,041,056$ \\
\hline & Fuel(thousand\$/rev. ton-kmyear & 52 \\
\cline { 2 - 3 } & Aircraft(thousand \$/one a/c·year) & 0.048 \\
\hline \multirow{2}{*}{ Output number(thousand revenue ton-km) } & 23,925 \\
\hline & Output value(\$/tousand-km) & $6,139,839$ \\
\hline
\end{tabular}

These elements of input, prices, and products were divided by the average price of each element of input to derive a standard index and to estimate the production function.

\subsection{Findings and Significance}

$<$ Table $4>$ shows the estimation of Formulas (1), (8a), and (8b) using the data above. As shown in this table, most estimated factors were significant at $10 \%$ or higher and the adjusted coefficient of determination was also high. The first terms of estimated parameters for all elements of input were positive as anticipated and were statistically significant. The marginal abatement cost of greenhouse gas in the aviation industry can be shown as Formula (9) by converting Formula (8a). Based on the average of samples, the marginal abatement cost of greenhouse gas in the aviation industry was 0.123 and was significant at $1 \%$. This factor means that the average cost of reducing 1 ton of greenhouse gas emission from aviation transportation is USD \$123.

$$
\lambda_{2}=\left[\alpha_{x}+\beta_{x x} \ln Z+\sum_{i} \gamma_{\xi} \ln X_{i}\right] /\left[\frac{Z}{P_{Q}\left(1-\frac{1}{n}\right) Q}\right]
$$


Table 4.

Results of Estimation for Production Ffunction

\begin{tabular}{|c|c|c|c|}
\hline Parameters & Estimates & Standard Error of Mean & t-value \\
\hline & 15.706 & 0.021 & $742.533^{* * *}$ \\
\hline & 0.793 & 0.020 & $40.322 * * *$ \\
\hline & 0.117 & 0.003 & $40.430^{* * *}$ \\
\hline & 0.109 & 0.003 & $31.807 * * *$ \\
\hline & 0.354 & 0.004 & $85.331^{* * *}$ \\
\hline & -0.058 & 0.003 & $-1.656^{*}$ \\
\hline & -0.006 & 0.011 & -0.580 \\
\hline & 0.013 & 0.014 & 0.957 \\
\hline & 0.068 & 0.016 & $4.198 * * *$ \\
\hline & 0.054 & 0.011 & $5.086 * * *$ \\
\hline & 0.013 & 0.013 & 1.012 \\
\hline & -0.081 & 0.012 & $-6.749 * * *$ \\
\hline & -0.043 & 0.009 & $-4.766^{* * *}$ \\
\hline & -0.015 & 0.010 & -1.592 \\
\hline & 0.039 & 0.013 & $3.080 * * *$ \\
\hline & 0.123 & 0.020 & $6.152 * * *$ \\
\hline statistics & \multicolumn{2}{|c|}{ Adj. $R^{2}$} & D-W statistics \\
\hline & \multicolumn{2}{|c|}{0.957} & \\
\hline
\end{tabular}

As shown in $<$ Fig $4>$, airlines would emit greenhouse gas until their marginal abatement cost reaches 0 without the government's environmental regulations. If the government imposes of environmental tax on per unit of greenhouse gas emission, the airlines would decide whether to emit greenhouse gases and pay the duties or to use voluntary measures to reduce greenhouse gas emissions. The marginal abatement cost of greenhouse gas for the estimated average of samples used in this study was approx. USD 123. If the government imposes the environmental tax on the airlines' greenhouse gas emissions and it exceeds USD 123 per ton, the airlines would save and be able to voluntarily reduce greenhouse gas until their marginal abatement cost is equal to the environmental tax. The amount to be paid for the environmental tax is larger than the profit 
earned from each unit of greenhouse gas emission. As the marginal abatement cost increases with lower greenhouse gas emission, the airlines would reduce their marginal abatement cost until it is equal to the environmental tax. On the other hand, if the environmental tax is less than USD 123 per ton, it would be more profitable to pay it instead of reducing greenhouse gas emission.

If the government chooses to regulate the total amount of emission, for example by emission trading, the marginal abatement cost of greenhouse gas would still be an important factor to decide the price of emission rights. Based on these results of estimation, the marginal abatement cost curve shown in $<$ Fig $4>$ was derived. As shown here, the marginal abatement cost curve of greenhouse gas emission in the aviation industry drew a declivity as the amount of emission increased. This means that the marginal abatement cost of greenhouse gas increases as greenhouse gas decreases.

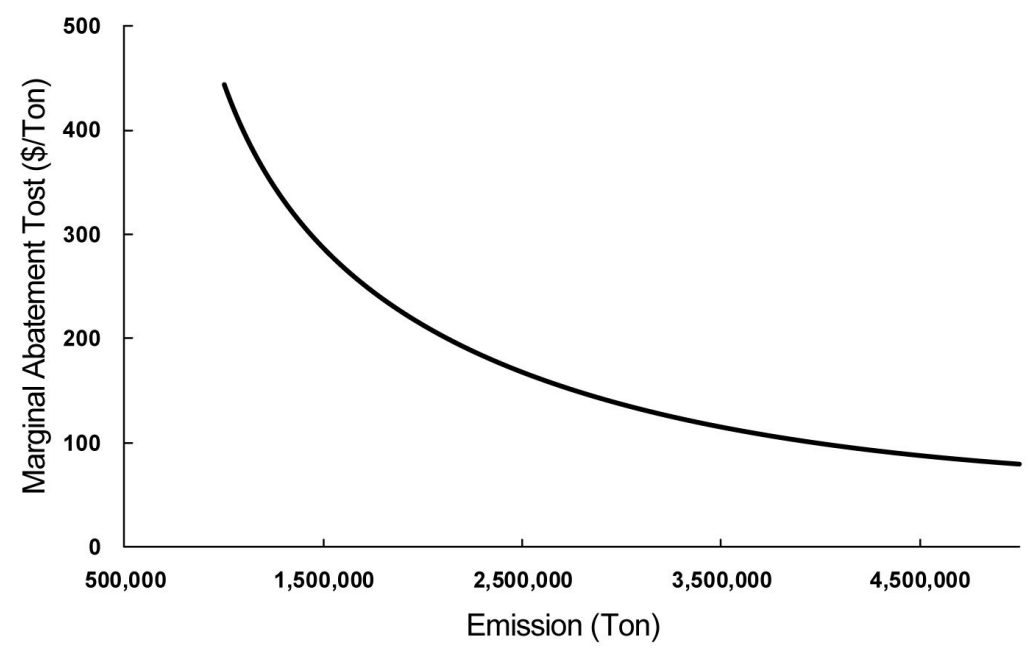

Figure 4.

Marginal Abatement Cost Curve of Greenhouse Gas from Airplanes

The appropriate amount of environmental tax can be derived by finding the intersection between this marginal abatement cost curve of greenhouse gas and the marginal damage curve based on the environmental damage caused by greenhouse gas emission. The appropriate amount of environmental tax is determined by the marginal damage curve. As mentioned above, if the optimal amount of environmental tax is higher than the current marginal abatement costs of the airlines, the airlines would voluntarily reduce greenhouse gas emission until their marginal abatement costs are equal to the amount of environmental tax. 


\section{Summary and Conclusion}

This study estimated greenhouse gas emission from aviation transportation and sought systems that can manage it based on the IPCC Guidelines to prepare for greenhouse gas regulations on international airlines. For this purpose, policies to reduce greenhouse gas emission from aviation transportation were developed based on international agreements and the cases of advanced countries, and the marginal abatement cost and greenhouse gas reduction measures were derived for the effective execution of these policies. As a result of estimating greenhouse gas emission from aviation transportation based on the IPCC Guidelines, it was found that there is an average increase of 3.9\% and $12.9 \%$ for domestic and international flights, indicating that it is urgent for us to prepare for global greenhouse gas regulations.

Also, greenhouse gas emission from airplanes is expected to increase by an average of $3.0 \%$ for high growth and an average of $1.3 \%$ for low growth for domestic routes and by an average of $5.2 \%$ for high growth and an average of 3.9\% for low growth for international routes, showing that emissions are expected to increase more rapidly for international flights. These prospects indicate that the amount of emission would exceed BAU (Business as Usual), which is the baseline of possible reduction of emission, by 2025 without any policies to reduce greenhouse gas emission from aviation transportation. BAU could also be used to rate the effectiveness of reduction policies. The estimated marginal abatement cost of greenhouse gas from airplanes was approx. USD 123, and this amount could be used to decide the price of emission rights and the amount of carbon tax and it could be referred to when distributing incentives for voluntary agreement. Based on the above results, this study suggested measures to reduce greenhouse gas and manage statistics on greenhouse gas emission for aviation transportation. The measures to reduce greenhouse gas emission for aviation transportation were classified into four types: voluntary agreement, international collaboration, greenhouse gas reduction technology and operation process development, and application of emission trading and carbon tax.

Five conditions need to be met for voluntary agreement to succeed. Firstly, programs and objectives between the government and companies need to be shared (motivation). Secondly, a partnerships must be formed by developing programs through collaboration. Thirdly, appropriate policy measures for each industry must be put into place (effectiveness). Fourthly, a market mechanism that is compatible with every industry must be utilized (economics), and lastly, it is learning, evaluating results, and improving programs (accountability and flexibility). International collaboration measures have to include, organizing a committee of East Asian governments to respond to the EU and develop collaborative greenhouse gas emission reduction measures for the aviation industry of Northeast Asia, and establishing a government committee for the statistics. It has to build and operate greenhouse gas information and statistics systems, and third, it is reporting and sharing results. Furthermore, for the development of greenhouse gas reduction technology and operation processes, we proposed technologies, standards, and ATM operations for engines and designs, as well as the necessities of sustainable management by developing technologies through $R \& D$, build and operating an efficient monitoring system. For market-based policies, emission trading 
and carbon tax policies, the most frequently used policies for greenhouse gas reduction, were examined to discuss their preparation, operation, and evaluation in the aviation industry. In addition, greenhouse gas emission statistics management measures were proposed for aviation transportation, along with subsequent legal regulations. In order to respond to the rapid changes of international trends with greenhouse gas, we need systemized response strategies. This study provided information on current emission, potential reduction, and management measures.

\section{References}

CE Delf. 2006. Aviation and maritime transport in a post 2012 climate policy regime.

ICAO. 2007. Environmental Report.

IPCC. 2006. IPCC guideline.

Graichen, Jakob and Bernd Gugele. 2006. Greenhouse Gas Emissions from Aviation. ETC/ACC Technical Paper.

Jamin, Solomon, Andereas Schäfer, Moshe E. Ben-Akiva, and Ian A. Waitz. 2004. Aviation emissions and abatement policies in the United States: a city-pair analysis. Transportation Research Part D. 9: 259 317.

Lee, Joosung J., Ian A. Waitz, B. Y. Kim, Gregg G. Fleming, Lourdes Maurice, and Curtis A. Holsclaw. 2007. System for assessing Aviation's Global Emissions (SAGE), Part 2: Uncertainty assessment. Transportation Research Part D. 12: 381 395.

Mayor, Karen and Richard S.J. Tol. 2007. The impact of the UK aviation tax on carbon dioxide emissions and visitor numbers. Transport Policy. 14: 507 513.

Pittman, Russel W. 1981. Issue in Pollution Control: Interplant Cost Diffrences and Economies of Scale. Land Economics. 125 143.

Scheelhaase, Janina D. and Wolfgang G. Grimme. 2007. Emissions trading for international aviation -an estimation of the economic impact on selected European airlines. Journal of Air Transport Management. 13:253 263.

UNFCCC. 2003. Compilation of data on emissions from international aviation. FCCC/TP/2003/3 14.

UNFCCC. 1999. Emissions resulting from fuel used for international transportation. FCCC/ SBSTA/1999/INF.4 11.

UNFCCC. 2003. Emissions resulting from fuel used in international aviation and maritime transportation. FCCC/SBSTA/2003/INF.3 14.

UNFCCC. 2004. Methodological issues relating to emissions from international aviation and maritime transport. FCCC/SBSTA/2004/INF.5 27.

UNFCCC. 1999. Methods used to collect Data, Estimate and Report Emissions from International Bunker Fuels. 12. 